\title{
EXPLOITATION DIDACTIQUE D'UNE PIÈCE DE THÉÂTRE EN CLASSE DE FLE - APPRENDRE LE FRANÇAIS PAR LE THÉÂTRE
}

\author{
Ileana Mihaela Chirițescu*, ORCID ID: 0000-0002-2272-7580 \\ University of Craiova, 13, A.I. Cuza st., Craiova, Romania \\ *Corresponding author: Ileana Mihaela Chirițescu, mihaela.chiritescu@edu.ucv.ro
}

Received: 11.28 .2021

Accepted: 01.22.2022

\begin{abstract}
Learning French through theater is one of the modern methods of learning, methods that are put at the service of a new didactics of teaching French. Theater performances can be a real motivation for students when used as a teaching aid for foreign language courses. Used as a teaching tool, theater has the ability to raise awareness and encourage students, as well as the strength to bring them effectively closer to the roots of the language studied. Theater means symbols that include characters, makeup, costumes, music, colors, soundtrack, painting. According to his vision, each person has an illusion about the world of theater performance - poetic, sentimental, cheerful, sad or melancholic illusion. With the help of all the artistic processes they have learned and which are at their disposal, each spectator and each actor transforms, in one way or another, into authors of the show, whose mission is to faithfully reproduce his own kind of illusion.
\end{abstract}

Keywords: théâtre, méthodes modernes d'enseignement, symboles, liberté.

Rezumat. Învățarea limbii franceze prin teatru reprezintă una dintre metodele moderne de învățare, metode care sunt puse în slujba unei noi didactici a predării limbii franceze. Spectacolele de teatru pot fi o motivație reală pentru studenți atunci când sunt folosite ca suport didactic pentru cursurile de limbi străine. Folosit ca instrument didactic, teatrul are capacitatea de a sensibiliza și de a încuraja studenții, precum și puterea de a-i apropia de rădăcinile limbii studiate. Teatrul presupune simboluri care includ personaje, machiaj, costume, muzică, culori, coloană sonoră, pictură. Conform viziunii sale, fiecare persoană are o iluzie despre lumea spectacolului teatral - iluzie poetică, sentimentală, veselă, tristă sau melancolică. Cu ajutorul tuturor procedeelor artistice pe care le-au învățat și care le stau la dispoziție, fiecare spectator și fiecare actor se transformă, într-un fel sau altul, în autori ai spectacolului, a căror misiune este de a reproduce cu fidelitate propriul tip de iluzie.

Cuvinte-cheie: teatru, metode moderne de predare, simboluri, libertate. 


\section{Introduction}

Le professeur est celui qui fait une classe de langue française attractive ou ennuyeuse et pour cela il doit utiliser toutes sortes de moyens, de méthodes et de matériels didactiques pour attirer les étudiants vers la connaissance d'une langue étrangère. Une autre raison et celle de souligner que les techniques audio-visuelles utilisées dans l'apprentissage de la langue française, peuvent faciliter le processus d'enseignement.

Apprendre le français par le théâtre fait partie des méthodes modernes d'apprentissage, méthodes qui sont mises au service d'une nouvelle didactique de l'enseignement du français.

Les pièces de théâtre peuvent représenter une réelle motivation pour les étudiants quand elles sont utilisées comme support didactique pour les cours de langue. Utilisé comme outil didactique, le théâtre, a la capacité de sensibiliser et d'inciter les étudiants, ainsi que la force de les rapprocher efficacement des racines de la langue étudiée.

D'une part, le théâtre représente un véritable support pour acquérir de nouvelles informations sur les traditions et l'histoire d'un peuple si son étude est intégrée dans les cours de français langue étrangère, et d'autre part, le théâtre encourage les étudiants à réfléchir aux idées qu'ils apprennent pour partager ces expériences avec d'autres futurs étudiants en vue de les encourager à se rapprocher de la langue étrangère en créant leurs propres stratégies de compréhension et de perception. Le théâtre signifie des symboles qui incluent des personnages, du maquillage, des costumes, des décors, de la musique, des couleurs, du fond sonore, de la peinture. Selon sa vision, chaque personne a une illusion sur le monde du spectacle de théâtre- une illusion poétique, sentimentale, gaie, triste ou mélancolique. À l'aide de tous les procédés artistiques qu'ils ont appris et qui se trouvent à leur disposition, chaque spectateur et chaque acteur se transforment, d'une manière ou d'une autre, en auteurs du spectacle, dont la mission est de reproduire fidèlement son propre type d'illusion.

\section{Exploitation didactique d'une pièce de théâtre en classe de FLE}

Le texte théâtral, comme tout texte littéraire, offre la possibilité de confronter l'apprenant avec la langue écrite, en lui offrant des ressources riches pour l'apprentissage du lexique, de l'orthographe et de la grammaire. Il constitue un outil adéquat pour l'acquisition des éléments particuliers pour les niveaux de langue. De même, on peut ajouter que l'enseignement du français présente la particularité de l'emploi des extraits provenant non seulement de la littérature française, mais aussi des littératures francophones. En enseignant la littérature, on enseigne aussi la culture et la civilisation d'une nation parce que, selon Le Petit Robert, la culture constitue l'ensemble des aspects intellectuels d'une nation et la civilisation renvoie à l'ensemble des phénomènes sociaux (religieux, moraux, esthétiques, scientifiques, techniques) communs à une grande société. La littérature sert de moyen d'acquisition d'une compétence linguistique et communicative et, quand ce n'est pas le cas, constitue une somme d'informations factuelles à apprendre [1].

Il est important de souligner que les textes littéraires authentiques présentent de nombreux avantages, mais y recourir, oblige l'enseignant de prendre des précautions pour obtenir les meilleurs résultats avec ses étudiants.

Utilisé comme nouvelle méthode d'enseignement pour les langues étrangères, même le texte dramatique peut avoir plusieurs interprétations selon la capacité de chaque enseignant à guider ses étudiants. Par exemple, les enseignantes sont plus proches de leurs 
étudiants, plus chaleureuses et plus positives dans leur vision de vie, toujours prêtes à donner le meilleur d'elles-mêmes au lieu de travail [2].

On ne pourra pas en faire le support principal du cours, on doit les intégrer dans un ensemble cohérent d'apprentissage et les choisir attentivement pour qu'ils répondent aux besoins des apprenants et pour qu'ils les attirent. Parfois, comme on l'a déjà dit, il est nécessaire d'adapter ces documents du point de vue de la longueur ou du contenu, pour qu'ils servent au but que l'on s'est proposé.

Dans le milieu universitaire, le rôle du tuteur est crucial. Le tutorat est considéré comme un processus de développement circulaire, où le dévouement est mutuel, le tuteur et les étudiants bénéficient également, de ce type de collaboration [3].

Par exemple, pour les classes de philologie ou de sciences humaines et sociales, l'enseignant peut introduire un cours optionnel ayant comme thème un sujet tiré de la littérature française: poésie, théâtre ou roman et alors, le temps sera suffisant pour que le professeur parcoure les étapes et les démarches proposées.

Victor Hugo disait que le théâtre n'est pas le pays du réel : il y a des arbres en carton, des palais de toile, un ciel de haillons, des diamants de verre, de l'or de clinquant, du fard sur la pêche, du rouge sur la joue, un soleil qui sort de dessous la terre. C'est le pays du vrai : il y a des cœurs humains dans les coulisses, des cœurs humains dans la salle, des cœurs humains sur la scène [4].

Le théâtre signifie illusion, les gens le comprennent selon leurs propres perceptions, leur expérience philologique, leur imagination, ou la façon dont ils perçoivent le monde en général.

Le théâtre est fait pour apprendre aux gens qu'il y a autre chose que ce qui se passe autour d'eux, que ce qu'ils croient voir ou entendre, qu'il y a un envers à ce qu'ils croient l'endroit des choses et des êtres, pour les révéler à eux-mêmes, pour leur faire deviner qu'ils ont un esprit et une âme immortels [5].

Le théâtre encourage les apprenants à utiliser leur imagination et leur sensibilité. Ils doivent percevoir une expérience à travers les rôles qu'ils interprètent, qui diffèrent de leur personnalité. Dans ces pièces, ils ont la possibilité de comprendre et d'inter-actionner avec les sentiments des autres. Cela développe la capacité des étudiants d'emphatiser, parce qu'ils apprennent de voir autour d'eux. Donc, le théâtre peut être utilisé comme un outil éducationnel qui développe les qualités morales et sociales de l'apprenant.

Par exemple, tout comme la déesse de la sagesse dans la mythologie grecque, Athéna, qui a pris la forme d'un vieillard sage pour se faire comprendre, de même le théâtre a la capacité de transfigurer les attitudes de ceux qui le jouent [6].

Le théâtre a charge de représenter les mouvements de l'âme, de l'esprit, du monde, de l'histoire [7].

Le théâtre étant un art public, exerce une fonction publique. Sa fonction visuelle est la fonction la plus importante du théâtre. Le cerveau a la capacité de percevoir la diversité qu'il filtre jusqu'à ce qu'il se familiarise avec les images qu'il voit et qu'il corrèle avec d'autres déjà connues [8].

C'est le public qui fait du théâtre un véritable forum politique, qui prend possession de la pièce et qui l'actualise, aussi bien dans le sens premier du terme, d'un passage à l'acte, que dans celui, plus récent, d'une mise à jour, attribuant ainsi à la pièce une portée aussi inédite qu'inattendue [9]. 
Le théâtre ne politise pas au sens propre du terme. Il met une loupe sur des enjeux, il permet de voir ce que, dans la vie réelle, n'est pas visible et il laisse penser aux contradictions de l'humain. La politique se concentre sur des contradictions.

L'éducation comprend toutes les influences qui ont lieu en dehors de l'école ou à travers des activités optionnelles ou facultatives [10].

Le théâtre met en évidence ces contradictions: la haine et l'amour, la liberté et la soumission, l'acceptation et le refus, et pourquoi pas, la vie et la mort. Au milieu scolaire, la politisation du théâtre doit être entendue comme motivation des élèves ou des étudiants de changer leur statut d'observateurs passifs en participants actifs aux activités de l'école et aux activités sociales aussi.

Donc, le théâtre peut créer des conditions où les apprenants peuvent faire des découvertes, où ils peuvent s'analyser comme personnes, où ils peuvent changer leur manière d'écouter et d'entendre les enseignants, où ils découvrent que les paroles ont des sens différents et que les gens sont différents. Par le théâtre, l'intelligence des étudiants va se développer par des procédés logico-déductifs. De plus, il aidera le professeur à établir les règles de la communication selon lesquelles on met en relation ce sur quoi l'on parle et ce que l'on en dit.

Il existe plusieurs circonstances pour former et informer les jeunes. La presse et les autres facteurs: l'église, l'école, la famille, les institutions culturelles doivent agir simultanément, en imbriquant leurs fonctions, et cela doit être fait pas du tout indépendamment ou de manière isolée. Il est important qu'entre ces environnements éducatifs s'établissent des relations de réciprocité et de cohérence et pas de relations de concurrence [11].

L'unique chose qui peut empêcher les apprenants sur leur voie vers le théâtre c'est la difficulté de lire. Ils trouvent, en général, beaucoup de mots inconnus et les structures grammaticales sont, souvent, trop compliquées, des facteurs qui mettent des barrières dans la compréhension d'une pièce. C'est pour cela qu'ils doivent commencer avec des lectures faciles pour ne pas être obligés à s'arrêter et à chercher tout le temps des mots dans le dictionnaire, fait qui les conduit à ne plus comprendre ce qu'ils ont lu. Les apprenants doivent sentir le plaisir de lire mais aussi voir l'utilité de la lecture. Les recherches ont démontré que les étudiants qui lisent en français se développent plus dans toutes les zones du processus d'apprentissage de cette langue que ceux qui ne le font pas.

Le but de l'éducation en ce qui concerne les valeurs est représenté par l'accès de l'être humain à l'autonomie et à la compétence professionnelle [12].

Lire c'est mettre en relation, c'est déchiffrer dans la linéarité. Gilles Aillaud parlait de l'existence des pièces qui ne sont pas à représenter mais à lire. Un lecteur actif peut intervenir dans le texte et participer à son élaboration, il peut arrêter le temps de l'action, fait ce qu'un spectateur ne peut pas faire, parce que la scène a la possibilité de compléter le texte, le redoubler, le superposer et même le nier. Alors, le théâtre devient art total et totalitaire.

On ne peut pas concevoir la compréhension d'une pièce de théâtre sans la lire correctement.

Les changements se font sur la base des visions innovantes, et le progrès ne se réalise pas en respectant les traditions, mais, au contraire, en défiant les sentiers battus [13].

Pour travailler sur le texte d'une pièce de théâtre, il faut le connaître et l'apprivoiser. Le premier pas et de lire plusieurs fois la pièce en entier et d'une seule traite, fait qui aura des bénéfices aussi sur l'acquisition de la langue. 
Pour notre activité on a choisi la pièce de théâtre "Le malade imaginaire ", acte III, scène 10écrite par Molière.

https://www.youtube.com/watch?v=s3qoPUFu07E

\section{Le déroulement des activités}

Niveau : B1

Durée : 50 min

Objectifs : s'exprimer à l'oral, analyser, enrichir le lexique, l'orthographe, les structures grammaticales, le jeu de scène.

Matériel : l'enregistrement de la pièce, la fiche avec les répliques de la pièce de théâtre, scène, décor créé par les étudiants.

Molière, de son vrai nom, Jean-Baptiste Poquelin, a été dramaturge, metteur en scène et acteur. Son génie créateur a marqué toute la dramaturgie française et universelle. Il a mis à jour dans une manière magistrale les défauts de l'espèce humaine : l'égoïsme, l'injustice, l'hypocrisie, l'hypocondrie, l'avarice. Son héritage artistique est impressionnant et les thèmes et les moyens artistiques sont actuels et modernes. Il a trouvé son fin, interprétant le rôle d'un malade imaginaire, le 12 février 1673.

https://fr.wikipedia.org/wiki/Moli\%C3\%A8re

\section{Activité 1}

Le professeur propose aux étudiants d'apprendre le français d'une manière tout à fait ludique, ayant comme support une pièce de théâtre, une comédie d'après Molière. Premièrement, le professeur discute avec ses étudiants sur la personnalité de ce grand dramaturge français, sur ses chefs d'œuvres et sur le fait qu'il a été aussi metteur en scène et acteur.

- Qui a été Molière?

„Molière a été un grand créateur des formes dramatiques, il a interprété le rôle principal de la plupart de ses pièces. Molière a exploité les diverses ressources du comique : verbal, gestuel et visuel, de situation et il a pratiqué tous les genres de comédie, de la farce à la comédie de caractère."

- Combien d'œuvres a-t-il écrit ? Quelles sont les plus importantes?

„Molière a créé environ trente comédies en vers ou en prose. Les comédies les plus appréciées sont : L'Avare, Le Bourgeois Gentilhomme, Les précieuses ridicules, Le Misanthrope, Le Tartuffe, Le Malade Imaginaire.

\section{Activité 2}

On fait une première lecture de la pièce jusqu'à la dernière phrase : Je viendrai... pour comprendre globalement la pièce.

Le professeur corrige les erreurs de prononciation et explique les mots inconnus en aidant ses étudiants à se fixer le lexique, l'orthographe et la structure grammaticale. Les étudiants répètent la prononciation correcte des répliques et lisent plusieurs fois la pièce en entier.

- Quels sont les personnages?

„Les personnages sont : Argan, le malade imaginaire, Toinette, sa servante (déguisée en médecin) et Béralde, le frère d'Argan."

- Où se passe la scène?

„La scène se passe dans la chambre d'Argan." 
- Est-ce que vous pouvez faire un court résumé de cet acte?

„Bref, Argan, un riche bourgeois, se croit malade. Le «héros» de la pièce est entouré des médecins et s'invente toutes sortes de maladies. Béralde, son frère, tente de lui parler de ses maladies imaginaires et lui conseille de se méfier des médecins. C'est finalement Toinette, la servante, qui se déguise en médecin pour duper son maître."

\section{Activité 3}

Le professeur distribue les rôles et encouragent les étudiants à se bouger, à jouer, à se connaître, à travailler en équipe. Ils doivent s'impliquer du point de vue émotionnel, d'entrer dans la peau des personnages, de travailler en harmonie les uns avec les autres ainsi que les étudiants changent leur statut d'observateurs passifs en participants actifs.

Les activités qui supposent des jeux de rôle, sont très appréciées par les étudiants. Pour moi, en qualité de professeur de français, les pièces de théâtre ont toujours été une bonne occasion de sortir de la routine pédagogique.

\section{Conclusions}

En conclusion, une pièce de théâtre se transforme dans un manuel de grammaire et d'orthographe, dans une mine de vocabulaire, de conjugaison et dans un facteur de communication. Le théâtre fait le lien entre réalité et l'illusion. Les étudiants adorent regarder des pièces de théâtre dont ils pourront ensuite discuter en français. Stimuler les étudiants à se rapprocher de la langue française à travers une pièce de théâtre, fait partie de nouvelles méthodes didactiques. Visionnées sur un vidéoprojecteur ou dans la salle des spectacles, les pièces de théâtre sont un bon moyen de sortir du quotidien et de regarder le monde d'une autre perspective.

\section{Notes:}

1. Windmüller Florence. Le français dans le monde, Revue de la Fédération internationale des professeurs de français, no. 348, nov-dec, 2006.

2. Bărbuceanu Costina Denisa. Athena rising? Mentoring in Higher Education, publié dans Revue des Sciences Politiques, Universitaria Publishing House, no. 62, 2019., p. 46.

3. Bărbuceanu Costina Denisa. Athena rising? Mentoring in Higher Education, publié dans Revue des Sciences Politiques, UniversitariaPublishing House, no. 62, 2019, p. 46.

4. Hugo Victor. Océan- Tas de pierre III, 1830-1833, A. Michel, Paris, 1942.

5. Jouvet Louis. Témoignages sur le théâtre, Étude (poche), Champs Arts, juin 2009.

6. Bărbuceanu Costina Denisa. Athena rising? Mentoring in Higher Education, publié dans Revue des Sciences Politiques, Universitaria Publishing House, no. 62, 2019, p. 46.

7. Mnouchkine Ariane. People/Premiere.fr, le 25 juin 2017, http://www.premiere.fr/Star/Ariane-MNOUCHKINE.

8. Barbuceanu Costina Denisa. Visual teaching- Usingdigitalisedmaterial to engage ESP students, publié dans Revue des Sciences Politiques, UniversitariaPublishing House, no. 67, 2020, p. 38.

9. Darlow Mark et Yann Robert. Laya-L'ami des lois, Édition présentée, établie et annotée, The Modern HumanitiesResearch Association, Londres, 2011.

10. Păunescu Anca. Mediatized word information. Education. Handling, dans Journal of Social Sciences, Technical University of Moldova, 2019, p. 29.

11. Păunescu Anca. Mediatized word information. Education. Handling, dans Journal of Social Sciences, Technical University of Moldova, 2019, p. 29.

12. Păunescu Anca. Mediatized word information. Education. Handling, dans Journal of Social Sciences, Technical University of Moldova, 2019, p. 30.

13. Păunescu Anca. Mediatized word information. Education. Handling, dans Journal of Social Sciences, Technical University of Moldova, 2019, p. 32. 


\section{References}

1. Bărbuceanu, Costina Denisa. Athena rising? Mentoring in Higher Education, publié dans Revue des Sciences Politiques, Universitaria Publishing House, no. 62, 2019.

2. Barbuceanu, Costina Denisa. Visual teaching- Usingdigitalisedmaterial to engage ESP students, publié dans Revue des Sciences Politiques, Universitaria Publishing House, no. 67, 2020.

3. Darlow Mark et Yann Robert. Laya-L'ami des lois, Édition présentée, établie et annotée, The modern humanitiesresearch association, londres, 2011.

4. Hugo Victor. Océan- Tas de pierre III, 1830 - 1833, A. Michel, Paris, 1942.

5. Jouvet Louis. Témoignages sur le théâtre, Étude (poche), Champs Arts, juin 2009.

6. Mnouchkine Ariane. People/Premiere.fr, le 25 juin 2017, http://www.premiere.fr/Star/Ariane-MNOUCHKINE

7. Moliere. Le malade imaginaire, Éditions Larousse, Paris, France, 2008.

8. Păunescu Anca. Mediatized word information. Education. Handling, dans Journal of Social Sciences, Technical University of Moldova, 2019.

9. Windmüller Florence. Le français dans le monde, Revue de la Fédération internationale des professeurs de français,no. 348, nov-dec, 2006.

10. https://www.youtube.com/watch?v=s3qoPUFu07E

11. https://fr.wikipedia.org/wiki/Moli\%C3\%A8re 